\title{
Theory of Differentlal Line Broadening in Coupled Spln Systems in Macromolecules and Molecular Aggregates. Applications to the Study of Micellar Systems
}

\author{
Lian-Pin Hwang, ${ }^{*}$ Pei-Lein Wang, \\ Department of Chemistry, National Taiwan University, Taipei, Taiwan, ROC, and Institute of Atomic and \\ Molecular Science, Academia Sinica, Nankang, Taipei, Taiwan, ROC \\ and Tuck C. Wong* \\ Department of Chemistry, University of Missouri, Columbia, Missouri 65211 (Received: July 9, 1987; \\ In Final Form: February 19, 1988)
}

\begin{abstract}
The theory of differential line broadening (DLB) in spin-coupled multiplets in molecular systems where there is a slow isotropic overall reorientation and a fast internal motion has been derived on the basis of the solution of the stochastic Liouville equation. The most prominent example is the proton-coupled methyl ${ }^{13} \mathrm{C}$ multiplet. From the line-width differences between various components of the multiplet, the correlation time for the slow overall reorientation and the chemical shift anisotropy can be determined. Applications to the study of the molecular dynamics of the sodium deoxycholate/water micellar system and a viscoelastic micellar system are illustrated. The results show that the DLB effect is a useful tool complementary to multifield relaxation time and nuclear Overhauser effect studies in the investigation of molecular dynamics.
\end{abstract}

\section{Introduction}

Differential line broadening (DLB) of nuclear magnetic resonance (NMR) lines in coupled spin $1 / 2$-spin $1 / 2$ systems was first discussed by Shimizu many years ago. ${ }^{1}$ The difference in the spin-spin relaxation times of different components in the multiplet can be attributed to the interference between the dipole-dipole (DD) and the chemical shift anisotropy (CSA) relaxation mechanisms. Such effects has since been observed in ${ }^{19} \mathrm{~F}-1{ }^{1} \mathrm{H}$ system, ${ }^{2}$ in ${ }^{31} \mathrm{P}-{ }^{19} \mathrm{~F}$ systems, ${ }^{3,4}$ in ${ }^{15} \mathrm{~N}-1 \mathrm{H}$ systems, ${ }^{5}$ in ${ }^{13} \mathrm{C}-{ }^{1} \mathrm{H}$ systems, ${ }^{6,7}$ in a ${ }^{31} \mathrm{P}-1 \mathrm{H}$ system, ${ }^{8}$ and in a ${ }^{1} \mathrm{H}^{1}{ }^{1} \mathrm{H}$ system. ${ }^{9} \mathrm{~A}$ detailed review of relaxation in coupled spin systems has been presented by Vold and Vold..$^{10}$ Farrar et al. ${ }^{7}$ have recently outlined the conditions under which the DLB effect is likely to be observed.

Recently, the DLB effect in proton-coupled methyl ${ }^{13} \mathrm{C}$ multiplets in two types of systems-micellar aggregates and methyl ${ }^{13} \mathrm{C}$-enriched transfer RNA-has been observed in our laboratory. ${ }^{11}$ In both of these systems, the dynamics of the molecules has to be described by more than one motion. Frequently, a model that consists of a slow $\left(\omega_{0} \tau_{c} \gtrsim^{+} 1\right)$ overall isotropic molecular (or aggregate) reorientation and a fast $\left(\omega_{0} \tau_{c} \ll 1\right)$ internal motion for the segment of the molecule of interest ${ }^{12,13}$ is applicable to these systems. In this paper, a derivation of this DLB effect in terms of such a dynamic model based on the solution of the stochastic Liouville equation (SLE) is presented. The line-width differences for a viscoelastic micellar system and for the sodium deoxycholate/water system are combined with magnetic field dependent

(1) Shimizu, H. J. Chem. Phys. 1964, 40, 3357.

(2) Mackor, E. L.; MacLean, C. Prog. NMR Spectrosc. 1967, 3, 129.

(3) Withers, S. G.; Madsen, N. B.; Sykes, B. D. J. Magn. Reson. 1985, 61,545 .

(4) Farrar, T. C.; Quintero-Arcaya, R. A. Chem. Phys. Lett. 1985, 122,

41. Farrar, T. C.; Ouintero-Arcaya, R. A. J. Phys. Chem. 1987, 91, 3224.

(5) Rueterjans, H.; Kaun, E.; Hull, W. E.; Limbach, H. H. Nucleic Acids Res. 1982, 10, 7027. Gueron, M.; Leroy, J. L.; Griffey, R. H. J. Am. Chem. Soc. 1984, 105, 7262 .

(6) Macura, S.: Brown, L. R. J. Magn. Reson. 1985, 62, 328.

(7) Farrar, T. C.; Adams, B. R.; Grey, G. C.; Quintero-Arcaya, R. A.; Zuo, Q. J. Am. Chem. Soc. 1986, 108, 8190 .

(8) Lunsford, J. H.; Rothwell, W. P.; Shen, W. J. Am. Chem. Soc. 1985, 107,1540 .

(9) Anet, F. A. L. J. Am. Chem. Soc. 1986, 108, 7102

(10) Vold, R. L.; Vold, R. R. Prog. NMR Spectrosc. 1978, 12, 79

(11) Gracz, H.; Agris, P., private communication.

(12) Wennerström, H.: Lindman, B.: Söderman, O.: Drakenberg, T.: Rosenholm; J. B. J. Am. Chem. Soc. 1979, 101, 6860.

(13) Lipari, G.; Szabo, A. J. Am. Chem. Soc. 1982, 104, 4546, 4559.
${ }^{13} \mathrm{C}$ spin-lattice relaxation time $\left(T_{1}\right)$ and nuclear Overhauser effect (NOE) measurements to deduce the dynamic and order parameters of the surfactants in these systems. The possible applications of the measurements of this DLB effect for the determination of the dynamics of molecules in those types of systemsmacromolecules and molecular aggregates-are also discussed.

\section{Theory}

For a complex molecule, internal rotation of the methyl group and overall reorientation of the molecule are present simultaneously. The spin relaxation of the methyl ${ }^{13} \mathrm{C}$ may be due to a composite of different magnetic interactions modulated by these motions. ${ }^{12,13}$ The magnetic interactions consist of mainly the chemical shift anisotropy (CSA) interactions and the dipolar interactions with protons. In the presence of a strong, externally applied magnetic field, $B_{0}$, the spin Hamiltonian is given by

$$
H=H_{0}+H_{\mathrm{d}}=H_{\mathrm{Z}}+H_{\mathrm{CSA}}+H_{\mathrm{d}}
$$

where

$$
H_{\mathrm{Z}}=-\omega_{\mathrm{C}} I_{\mathrm{Z}}-\omega_{\mathrm{H}} S_{\mathrm{Z}}+J I_{\mathrm{Z}} S_{\mathrm{Z}}
$$

It includes the Zeeman terms for ${ }^{13} \mathrm{C}$ ( $I$ spin) and protons ( $S$ spin) in a methyl group as well as the first-order correction of the spin-spin coupling between them. The interaction of chemical shift anisotropy is given by ${ }^{14}$

$H_{\mathrm{CSA}}=F D_{0,0}^{(2)}(\Omega) I_{\mathrm{Z}}-(3 / 8)^{1 / 2} F\left\{-D_{0,1}^{(2)}(\Omega) I_{+}+D_{0,-1}^{(2)}(\Omega) I_{-}\right\}$

where $F=2 / 3\left(\sigma_{\|}-\sigma_{\perp}\right) \omega_{I}$ in which $\sigma_{\|}$and $\sigma_{\perp}$ are the parallel and perpendicular principal values of the CSA tensor, which is assumed to have cylindrical symmetry in the present case. The principal axis of the CSA tensor is considered to be coincident with the $C_{3}$ axis of the methyl group, which is chosen as the molecule-fixed frame. The $D_{K, M}^{(L)}(\Omega)$ are Wigner rotation matrix elements ${ }^{15}$ that express the transformation from the molecule-fixed $\left(x^{\prime}, y^{\prime}, z\right)$ to the laboratory $(x, y, z)$ axis sytem, where $\Omega$ represents the Euler angles relating one axis system to the other. The dipolar Hamiltonian depends on the orientation $\Omega$ and the internal rotation coordinates $(\alpha, \beta, 0)$. Here $\alpha$ is the rotating angle of the methyl group, and $\beta$ is a fixed angle between the methyl $C_{3}$ axis and the

(14) Abragam, A. The Principles of Nuclear Magnetism; Oxford University Press: Oxford, 1961, Chapter 8.

(15) Brink, D. M.; Satchler, G. R. Angular Momentum; Oxford University Press: Oxford, 1968 
C-H direction. The dipolar interaction Hamiltonian may be written as the scalar product of two tensors, ${ }^{14,16}$ i.e.

$$
\begin{aligned}
H_{\mathrm{d}}(\Omega, \alpha)=\sum_{p, q} D_{q, 0}^{(2)}(\alpha, \beta, 0) D_{q, p}^{(2)}(\Omega) A_{p}= \\
\quad \sum_{p, q} \exp (i q \alpha) d_{q, 0}^{(2)}(\beta) D_{q, p}^{(2)}(\Omega) A_{p}
\end{aligned}
$$

where $d_{q, 0}^{(2)}(\beta)$ is the reduced Wigner rotational matrix element, and where for the dipolar interaction of nuclear $I$ and $S^{\prime}, A_{p}$ is given by

$$
\begin{gathered}
A_{2}=-D^{\prime} I_{+} S_{+}^{\prime} \quad A_{1}=D^{\prime}\left(I_{Z} S_{+}^{\prime}+I_{+} S_{Z}^{\prime}\right) \\
A_{0}=-(6)^{-1 / 2} D^{\prime}\left(4 I_{Z} S_{Z}^{\prime}-I_{+} S_{-}^{\prime}-I S_{+}^{\prime}\right) \\
A_{-1}=A_{1}{ }^{*} \quad A_{-2}=A_{2}{ }^{*}
\end{gathered}
$$

where $S^{\prime}=1 / 2$ denotes the spin quantum number for one proton spin only. $D^{\prime}=(3 / 2)^{1 / 2} \gamma_{\mathrm{I}} \gamma_{S^{\prime}} \hbar / r^{3}$, and $r$ is the bond length of $\mathrm{C}-\mathrm{H}$. Here the effects of cross relaxation among protons in the methyl group are not included in the treatment of dipolar relaxation between ${ }^{13} \mathrm{C}$ and ${ }^{1} \mathrm{H}$. In the present approach, we started from the stochastic Liouville equation (SLE) and considered the overall reorientation and internal rotation as independent motions, i.e.

$$
\dot{\rho}(\Omega, \alpha, t)=\left\{-i\left(\hat{L}_{0}+\hat{L}_{\mathrm{d}}\right)-\hat{\Gamma}_{\Omega}-\hat{\Gamma}_{\alpha}\right\} \rho(\Omega, \alpha, t)
$$

where $\rho$ is the spin density matrix in a state $(\Omega, \alpha) . \hat{\Gamma}_{\alpha}$ and $\hat{\Gamma}_{\Omega}$ are the diffusion operators for internal rotation and overall reorientation, respectively. $\hat{L}_{0}$ and $\hat{L}_{\mathrm{d}}$ are the Liouville operators corresponding to $H_{0}$ and $H_{\mathrm{d}}$, respectively. Since the internal rotational mode is considered a priori to be much faster than the overall reorientation, $\alpha$ is a fast variable for the internal motion, which may be considered to be independent of the overall reorientation. Thus, we may introduce a coarse graining in time such that the spin density is averaged over a period much longer than the correlation time of the internal rotation but still small compared to the correlation time of the overall reorientation. Then, we followed the approach of Hwang ${ }^{16}$ to reduce the SLE by averaging over the fast variable and treating the dipolar interaction as a perturbation. This then gives

$$
\dot{\sigma}(\Omega, t)=\left[-i\left(\hat{L}_{0}^{\prime}+\hat{L}_{\mathrm{d}}^{\prime}\right)-\hat{\Gamma}_{\Omega}-1 / T_{2}(\Omega)\right] \sigma(\Omega, t)
$$

where the reduced density matrix $\sigma$ is defined as the density matrix $\rho$ averaged over $\alpha$ isotropically. $\hat{L}_{\mathrm{d}}^{\prime}$ is the Liouville operator corresponding to the averaged dipolar Hamiltonian:

$$
H_{\mathrm{d}}^{\prime}=\left\langle H_{\mathrm{d}}(\Omega, \alpha)\right\rangle_{\alpha}=\mathrm{S}_{C_{3}} d_{0,0}^{(2)}(\beta) \sum_{p} D_{0, p}^{(2)}(\Omega) A_{p}
$$

$\mathrm{S}_{C_{3}}$ is the order parameter for the $C_{3}$ axis of the methyl group and is defined as

$$
\mathrm{S}_{C_{3}}=1 / 2\left\langle 3 \cos ^{2} \theta-1\right\rangle_{\alpha}
$$

where $\theta$ is the angle between the $C_{3}$ axis and the director of the aggregate. The averaging is over the fast internal motion $\alpha^{12,13}$ $\hat{L}_{0}^{\prime}$ is the Liouville operator corresponding to the averaged $H_{0}$ in which the CSA interaction is averaged by the fast motion $\alpha$. Thus, the molecular orientation-dependent spin-spin relaxation rate, at the instant of molecular orientation specified by the Euler angle $\Omega$, due to the modulation of the dipolar interaction by the internal rotation is given by

$$
1 / T_{2}(\Omega)=X+Y D_{0,0}^{(2)}(\Omega)
$$

where

$$
X=N \gamma_{C^{2}} \gamma_{\mathrm{H}^{2}} \hbar^{2} S^{\prime}\left(S^{\prime}+1\right) r^{-6}\left[2 \sin ^{2}(2 \beta) \tau_{1}+\sin ^{4}(\beta) \tau_{2}\right]
$$

$Y=N \gamma_{C^{2}} \gamma_{H^{2}} \hbar^{2} S^{\prime}\left(S^{\prime}+1\right) r^{-6}\left[\sin ^{2}(2 \beta) \tau_{1}-\sin ^{4}(\beta) \tau_{2}\right] / 4$

$N=3$ denotes the number of protons in a methyl group. The motional narrowing condition for internal rotation was invoked in the derivation. $\tau_{1}$ and $\tau_{2}$ are the correlation times for internal

(16) Hwang, L. P. Mol. Phys. 1983, 49, 1314. rotation, with $\tau_{2}=\tau_{1} / 4$ for the Brownian model and $\tau_{2}=\tau_{1}$ for $120^{\circ}$ jump model. The derivation is similar to the work of Donskaya in his treatment of $T_{1}$ process for proton-proton dipolar relaxation due to internal rotation. ${ }^{17}$ In the next section, $\tau_{\mathrm{c}}^{\mathrm{f}}$, the correlation time for the fast methyl reorientation is used instead of $\tau_{2}$. If the $C_{3}$ axis of the methyl group coincides with the director, then these two correlation times are equivalent.

The reduced stochastic equation may be solved by the eigenfunction expansion method of Freed et al.$^{18}$ in their treatment of slow-motion line shape. For three proton spins in a methyl group, there are eight possible spin states. They may be separated into a quartet $(S=3 / 2)$ and two doublets $(S=1 / 2) .{ }^{19}$ Therefore, the proton-coupled ${ }^{13} \mathrm{C}$ spectrum may be treated as a superposition of individual spectra from the coupling of ${ }^{13} \mathrm{C}$ with one $S=3 / 2$ spin and two $S=1 / 2$ spins separately. Furthermore, the motionally narrowed results of the reduced stochastic equation may be obtained by retaining the second-order terms in the interactions. The perturbation method for solving the SLE in the study of ESR line widths has previously been discussed in several works. ${ }^{18,20,21}$ Analogously, we may derive a modified motional narrowing line-width equation for proton-coupled ${ }^{13} \mathrm{C}$ spectra to include the effects of CSA and dipolar interactions as well as their cross effect by means of a perturbation analysis of the slow-motion equations. ${ }^{18}$ If overall reorientational diffusion is assumed to be isotropic and Brownian, the results may be expressed by

$$
1 / T_{2}\left(m_{\mathrm{s}}, \tau\right)=a+b m_{\mathrm{s}}+c m_{\mathrm{s}}^{2}
$$

where

$$
\begin{gathered}
a=a^{\prime}+X+\tau_{0}\left\{\left(S_{C_{3}}{ }^{2} F^{2}-Y^{2}\right) / 5+3 S_{C_{3}}{ }^{2} F^{2} U_{0} / 20+S(S+\right. \\
\left.1) D^{2}\left(P+U_{-} / 3+2 U_{+}\right) / 5\right\} \\
b=-(32 / 75)^{1 / 2} \tau_{0} S_{C_{3}} F D\left(1+3 U_{0} / 4\right) \\
c=(8 / 15) \tau_{0} D^{2}\left(1-3 P / 8+3 U_{0} / 4-U_{-} / 8-3 U_{+} / 4\right)
\end{gathered}
$$

with

$$
\begin{gathered}
D=\mathrm{S} D^{\prime} \quad P=\left[1+\left(\omega_{\mathrm{H}} \tau_{0}\right)^{2}\right]^{-1} \\
U_{0}=\left[1+\left(\omega_{\mathrm{C}} \tau_{0}\right)^{2}\right]^{-1} \\
U_{ \pm}=\left[1+\left(\omega_{\mathrm{C}} \pm \omega_{\mathrm{H}}\right)^{2} \tau_{0}{ }^{2}\right]^{-1}
\end{gathered}
$$

Here $\tau_{0}$ is the correlation time of the overall reorientational motion and is equivalent to $\tau_{c}^{\mathrm{s}}$ used in the subsequent sections. $m_{\mathrm{s}}$ is the magnetic spin quantum number with the total spin quantum number defined by $S$ as before. We also limit ourselves to the square term of $m_{\mathrm{s}}$ and neglect the small contribution from higher $m_{\mathrm{s}}$ terms. $a^{\prime}$ is the residual line width (in rad/s) that is attributable to spin-rotation interactions and other unspecified relaxation mechanisms such as long-range intramolecular or intermolecular dipole-dipole relaxation. The order parameter $S$ represents the order parameter for the $\mathrm{C}-\mathrm{H}$ interatomic vector. Usually, this order parameter is related to $S_{C_{3}}$ by the following relation:

$$
\mathrm{S}=\mathrm{S}_{C_{3}} d_{0,0}(2)(\beta)=\mathrm{S}_{C_{3}}\left(3 / 2 \cos ^{2} \beta-1 / 2\right)
$$

The product $F \mathrm{~S}_{\mathrm{C}_{3}}$ in eq 15 thus represents the residual CSA as a result of averaging by the fast motion, assuming cylindrical symmetry with respect to the $C_{3}$ axis of the methyl group.

It should be pointed out that the SLE approach used in this work is an alternative to the well-known Redfield approach, ${ }^{22}$ which has been used in several other studies relating to DLB. $4,5,7,23$

(17) Donskaya, I, S. Mol. Phys. 1975, 29, 1361

(18) Freed, J. H.; Bruno, G. V. Polnaszek, C. F. J. Phys. Chem. 1971, 75 3385. Polnaszek, C. F.; Bruno, G. V.; Freed, J. H. J. Chem. Phys. 1973, 58 3185

(19) Carrington, A.; McLachlan, A. D. Introduction to Magnetic Resonance; Harper and Row: New York, 1967; Chapter 4.

(20) Freed, J. H. J. Phys. Chem. 1974, 78, 1155

(21) Hwang, J. S.; Mason, R. P.; Hwang, L. P.; Freed, J. H. J. Phys. Chem. 1975, 79, 489.

(22) Redfield, A. G. Advances in Magnetic Resonance; Waugh, J. S., Ed.; Academic: New York, 1965; Vol. 1

(23) Prestegard, J. H.; Grant, D. M. J. Am. Chem. Soc. 1978, 100, 4664 
TABLE I: Line-Width Differences of the Methyl ${ }^{13} \mathrm{C}$ Multiplet in the $\boldsymbol{N}$-Methyl Group in CTAB in CTAB/Nasal Viscoelastic Solutions and the Slow Correlation Time and Chemical Shift Anisotropy Calculated from These line-Width Differences

\begin{tabular}{lcrccc}
\hline sample & $\begin{array}{c}\text { temp, } \\
{ }^{\circ} \mathrm{C}\end{array}$ & $\begin{array}{c}\Delta \nu(-1 / 2,1 / 2),{ }^{a} \\
\mathrm{~Hz}\end{array}$ & $\begin{array}{c}\Delta \nu\left({ }^{3} / 2,{ }^{1} / 2\right), \\
\mathrm{Hz}\end{array}$ & $\begin{array}{c}10^{6} \tau_{\mathrm{c}}{ }^{3},{ }^{b} \\
\mathrm{~s}\end{array}$ & $\begin{array}{c}\Delta \sigma,{ }^{b, c} \\
\mathrm{ppm}\end{array}$ \\
\hline I $^{d}$ & 38 & $10.0 \pm 2.5$ & & & \\
I & 42 & $7.5 \pm 1.0$ & $21.1 \pm 3.0$ & $2.2 \pm 0.5$ & $-75 \pm 18$ \\
I & 47 & $6.0 \pm 0.8$ & $20.4 \pm 2.0$ & $2.1+0.4$ & $-65+12$ \\
I & 52 & $4.6 \pm 0.5$ & $18.5 \pm 1.5$ & $1.8 \pm 0.25$ & $-57 \pm 7$ \\
I & 56 & $4.0 \pm 0.3$ & $15.0 \pm 1.0$ & $1.5 \pm 0.15$ & $-60 \pm 6$ \\
II d & 50 & $6.8 \pm 0.5$ & $23.8 \pm 1.0$ & $2.4 \pm 0.16$ & $-64 \pm 6$ \\
II & 55 & $6.0 \pm 0.5$ & $19.6 \pm 2.0$ & $2.0 \pm 0.3$ & $-67 \pm 10$
\end{tabular}

${ }^{a} \Delta \nu\left(m_{1}, m_{2}\right)$ is defined as $\Delta \nu_{1 / 2}\left(m_{1}\right)-\Delta \nu_{1 / 2}\left(m_{2}\right)$, and $\Delta \nu_{1 / 2}$ is the full width of the transition at half-height. All $\Delta \nu$ were measured at $B_{0}=$ $7.0 \mathrm{~T}$. ${ }^{b} \mathrm{The}$ calculations were based on $\mathrm{S}=0.037$ (and $\mathrm{S}_{\mathrm{C}_{3}}=0.11$ ) as determined from ${ }^{2} \mathrm{H}$ splittings in the liquid crystalline phases (see text). The order parameters are temperature and concentration dependent. However, these dependences over a narrow temperature and concentration range are quite small and were neglected in the present calculation. Since both the quadrupolar coupling constant for the C-D bond and the $\mathrm{C}-\mathrm{H}$ dipolar interaction have to be used in the calculation, the value of the ratio of these two parameters according to ref 51 was used. ${ }^{c} \Delta \sigma=\sigma_{l}-\sigma_{\perp}$. ${ }^{d}$ Sample I is $16.5 \mathrm{mM} \mathrm{CTAB}$ and $14.6 \mathrm{mM}$ Nasal in $\mathrm{D}_{2} \mathrm{O} / \mathrm{H}_{2} \mathrm{O}$; sample II is $17.0 \mathrm{mM}$ CTAB and $18.3 \mathrm{mM}$ Nasal in $\mathrm{D}_{2} \mathrm{O} / \mathrm{H}_{2} \mathrm{O}$.

\section{Experimental Section}

Hexadecyltrimethylammonium Bromide/Sodium Salicylate/ Water Viscoelastic Micelles. Viscoelastic micellar solutions ${ }^{24}$ were prepared by dissolving appropriate amounts of hexadecyltrimethylammonium bromide (CTAB) and sodium salicylate (Nasal) in a mixture of $\mathrm{H}_{2} \mathrm{O}$ and $\mathrm{D}_{2} \mathrm{O}(7: 3 \mathrm{v} / \mathrm{v})$, the latter being needed to provide the field-frequency lock in the NMR measurements. The concentrations of CTAB and Nasal were in the range of 13-20 $\mathrm{mM}$ and are listed in Table $\mathrm{I}$. Prior to the addition of Nasal, the viscosity of the dilute CTAB solution was very low, typical of dilute aqueous micellar solutions. Upon the addition of about equimolar of Nasal and rigorous mixing, viscoelastic behavior of the resulting solutions becomes obvious. ${ }^{24}$ CTAB (95\%) and Nasal (99\%) were purchased from Aldrich Chemicals. Both were used as received without further purification. Samples of "normal" micellar solutions of $\mathrm{CTAB}$ in $\mathrm{D}_{2} \mathrm{O}$ with and without a cosurfactant, pentanol, were also prepared for comparison. The ${ }^{13} \mathrm{C}$ line widths of the $\mathrm{N}\left(\mathrm{CH}_{3}\right)_{3}$ multiplets were measured for these solutions.

${ }^{13} \mathrm{C}$ NMR measurements on the viscoelastic micelles were made on a Nicolet NT-300 spectrometer operating at $75.4 \mathrm{MHz}$ and on a Bruker AM- 400 spectrometer operating at $100.5 \mathrm{MHz}$. Line-width measurements were made only at $75.4 \mathrm{MHz}$. In both cases, a $20-\mathrm{mm}$ probe was used. The $\pi / 2$ pulses for the two spectrometers are 45 and $28 \mu \mathrm{s}$, respectively. The coupled ${ }^{13} \mathrm{C}$ spectra were taken with the inversely gated decoupling scheme. The spin-lattice relaxation time, $T_{1}$, was measured by using the fast inversion recovery (FIRFT) method, ${ }^{25}$ where the delay time between acquisitions was set to 1-2 times the estimated $T_{1}$. NOE was measured by the "dynamic NOE" method. ${ }^{26} T_{1}$ was also obtained from such measurements. The $T_{1}$ values obtained from these two techniques are consistent with each other within experimental error.

Sodium Deoxycholate/Water Micelles. Sodium deoxycholate was purchased from Sigma Chemical Co. and was used as received without further purification. The sample used for this study was $75 \mathrm{mM}$ sodium deoxycholate in $0.1 \mathrm{M} \mathrm{Na}_{2} \mathrm{CO}_{3}$ solution in $\mathrm{D}_{2} \mathrm{O}$. For this system, ${ }^{13} \mathrm{C}$ measurements were made on a Nicolet NT-300 and Bruker AM-400, AM-300, MSL-200, and MSL-90 spectrometers. In the measurement on the NT-300 spectrometer, a $20-\mathrm{mm}$ probe was used. In other cases, $10-\mathrm{mm}$ probes were used.

(24) Ulmius, J.; Wennerström, H.; Johanson, L. B.-A.; Lindblom, G.; Gravsholt, S. J. Phys. Chem. 1979, 83, 2232.

(25) Canet, D.; Levy, G. C.; Peat, I. R. J. Magn. Reson. 1975, 18, 199. 327.

(26) Freeman, R.; Hill, H. D. W.; Kaptein, R. J. Magn. Reson. 1972, 7,

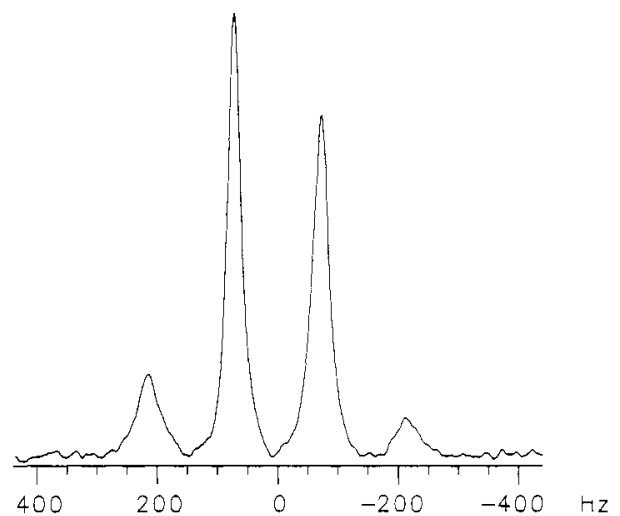

Figure 1. Proton-coupled ${ }^{13} \mathrm{C}$ spectrum of the $N$-methyl group of CTAB in a CTAB/Nasal viscoelastic micellar solution (sample I) at $52^{\circ} \mathrm{C}$. The spectrum was taken at $75.5 \mathrm{MHz}\left(B_{0}=7.0 \mathrm{~T}\right)$.

The experimental techniques used to obtain the coupled ${ }^{13} \mathrm{C}$ spectra, $T_{1}$, and NOE were similar to those utilized for the viscoelastic micellar system.

\section{Results}

Differential Line Broadening. CTAB/Nasal/Water Viscoelastic Micellar System. From the proton-coupled ${ }^{13} \mathrm{C}$ spectra of the CTAB/Nasal viscoelastic solutions, the line-width differences between the various components of the proton-coupled multiplet of the $N$-methyl carbon in CTAB was clearly observed (Figure 1). Since ${ }^{1} J_{\mathrm{CH}}$ is known to be positive, the downfield outer line in the quartet corresponds to the $m_{\mathrm{s}}=3 / 2$ proton spin state, and the downfield inner line corresponds to the $m_{\mathrm{s}}=1 / 2$ state, etc. The broadness and the marginal signal-to-noise ratio of the outer lines made the measurement of their line widths more difficult. In all cases, the line width of the upper-field outer line $\left(m_{\mathrm{s}}=-3 / 2\right)$ could not be measured to a satisfactory degree. However, the difference between the widths of the outer lines and those of the inner lines and the difference between the widths of the two inner lines are quite obvious. On the other hand, the multiplet for the terminal methyl group on the $\mathrm{C}_{16}$ chain of $\mathrm{CTAB}$ showed marginal DLB only at the lower end of the temperature range under study $\left(\approx 38-42^{\circ} \mathrm{C}\right)$. Apparently, the much smaller order parameter for the terminal methyl group and the expected smaller CSA of the $C$-methyl ${ }^{13} \mathrm{C}$ (as compared to $N$-methyl) ${ }^{27}$ rendered the DLB too small to be detected. Although the exact temperature range of which the CTAB/Nasal solutions exhibit viscoelastic properties has not been investigated in this study, it was observed that the solutions remained viscoelastic up to at least $56^{\circ} \mathrm{C}$, and DLB persisted up to this temperature. For the normal micellar solutions of $\mathrm{CTAB}$ in $\mathrm{D}_{2} \mathrm{O}$ with and without pentanol, no DLB was detected even at lowered temperature $\left(\sim 5^{\circ} \mathrm{C}\right)$. The line widths in general are affected by magnetic field inhomogeneity, temperature fluctuations in long-term signal averaging, and the necessary line broadening during data processing for the purpose of increasing the signal-to-noise ratio. For the $N$-methyl signals in the present case, the line widths are further affected by scalar relaxation due to the fast relaxation of the adjacent ${ }^{14} \mathrm{~N}$ nucleus and, in the coupled spectrum, by the unresolved long-range ${ }^{3} J_{\mathrm{CH}}$, which is estimated to be $3-4 \mathrm{~Hz}^{28}$ From the typical magnitude of ${ }^{15} \mathrm{~N}-{ }^{13} \mathrm{C}$ scalar coupling constants and the $T_{1}$ of ${ }^{14} \mathrm{~N}$ in similar systems, ${ }^{29}$ the scalar relaxation due to ${ }^{14} \mathrm{~N}$ is estimated to contribute approximately $2 \mathrm{~Hz}$ to the line widths. However, all the contributions described here contribute equally to all the components of the multiplet. Therefore in Table I, only the differences in the line widths are reported. The $m_{\mathrm{s}}$-dependent terms ( $b$ and $c$ in eq 13) can be directly deduced from these differences, while the $m_{\mathrm{s}}$-independent contributions are cancelled. ${ }^{30}$ It can easily be shown

(27) Veeman, W. S. Prog. NMR Spectrosc. 1984, 16, 193.

(28) Hansen, P. E. Prog. NMR Spectrosc. 1981, 14, 175.

(29) Henriksson, U.; Ödberg, L. Finn. Chem. Lett. 1982, 127.

(30) In fact, the a term for the quartet and the two doublets in the proton spin states is slightly different (eq 14). However, this difference is negligibly small for $\tau_{0} \sim 10^{-8} \mathrm{~s}$ or longer. 
TABLE II: ${ }^{13} \mathrm{C} T_{1}$ (in seconds) and NOE for the $N$-Methyl Group of CTAB in the CTAB/Nasal Viscoelastic Micellar Solutions at Two Magnetic Fields (7.0 and 9.4 T)

\begin{tabular}{ccccccc}
\hline & \multirow{2}{*}{$\begin{array}{c}\text { temp, } \\
\text { sample }\end{array}$} & \multicolumn{2}{c}{$7.0 \mathrm{~T}$} & & \multicolumn{2}{c}{$9.4 \mathrm{~T}$} \\
\cline { 5 - 6 } & ${ }^{\circ} \mathrm{C}$ & $T_{1}$ & $\eta$ & & $T_{1}$ & $\eta$ \\
\hline I & 42 & 0.619 & 1.27 & & 0.753 & 1.52 \\
I & 47 & 0.721 & 1.38 & 0.841 & 1.60 \\
I & 52 & 0.802 & 1.47 & 0.915 & 1.69 \\
II & 50 & 0.672 & 1.56 & & \\
II & 55 & 0.747 & 1.60 & &
\end{tabular}

${ }^{a}$ Samples I and II are as denoted in Table I.

TABLE III: Experimental ${ }^{13} \mathrm{C} T_{1}$ and NOE at Several Magnetic Fields for $C_{18}, C_{21}$, and $C_{19}$ of Sodium Deoxycholate in a $50 \mathrm{mM}$ Micellar Solution at $25{ }^{\circ} \mathrm{C}$

\begin{tabular}{cccc}
\hline $\begin{array}{c}\text { magn field } \\
B_{0}, \mathrm{~T}\end{array}$ & $\mathrm{C}_{\text {no. }}$ & $T_{1}, \mathrm{~s}$ & $\eta$ \\
\hline 9.4 & $\mathrm{C}_{18}$ & 0.84 & 1.12 \\
& $\mathrm{C}_{21}$ & 0.56 & 1.37 \\
& $\mathrm{C}_{19}$ & 0.76 & 1.10 \\
7.0 & $\mathrm{C}_{18}$ & 0.71 & 0.96 \\
& $\mathrm{C}_{21}$ & 0.50 & 1.18 \\
& $\mathrm{C}_{19}$ & 0.61 & 1.02 \\
4.7 & $\mathrm{C}_{18}$ & 0.51 & 0.74 \\
& $\mathrm{C}_{21}$ & 0.36 & 1.01 \\
& $\mathrm{C}_{19}$ & 0.39 & 0.78 \\
& $\mathrm{C}_{18}$ & $0.19^{a}$ & 0.71 \\
& $\mathrm{C}_{21}$ & $0.16^{a}$ & 0.91 \\
& $\mathrm{C}_{19}$ & $0.18^{a}$ & 0.75
\end{tabular}

${ }^{a}$ The null-point method was used in these measurements.

that the line-width difference between the $m_{\mathrm{s}}=-1 / 2$ and $m_{\mathrm{s}}=$ $1 / 2$ lines, $\Delta \nu(-1 / 2,1 / 2)$ (in hertz), is given by

$$
\pi \Delta \nu(-1 / 2,1 / 2)=1 / T_{2}(-1 / 2)-1 / T_{2}(1 / 2)=-b
$$

Similarly, $\Delta v(3 / 2,1 / 2)$ is given by

$$
\pi \Delta \nu(3 / 2,1 / 2)=b+2 c
$$

While the $b$ term arises from the interference between the DD and CSA relaxation mechanisms (eq 15), the $c$ term is dependent only on the DD relaxation (eq 16). For $\tau_{\mathrm{c}}^{\mathrm{s}} \sim 10^{-8} \mathrm{~s}$, the spectral densities, $U_{0}, U_{ \pm}$, and $P$ are negligibly small $(\sim 0.01)$ at $B_{0} \approx 7$ $\mathrm{T}$. Since the dipolar interaction between the ${ }^{13} \mathrm{C}$ and the protons can be easily estimated, the determination of the $b$ and $c$ terms provides a convenient way to obtain direct information on the values of $\tau_{\mathrm{c}}^{\mathrm{s}}$ and the CSA.

Sodium Deoxycholate/Water Micellar System. The magnitude of DLB for the methyl $\left(\mathrm{C}_{18}\right.$ and $\mathrm{C}_{21}{ }^{13} \mathrm{C}$ multiplets was an order of magnitude smaller than that observed in the $N$-methyl multiplet in CTAB in the viscoelastic micelles described in the preceding section. In fact, the line-width difference between the two inner lines, $\Delta \nu(-1 / 2,1 / 2)$, was too small to be measured. However, the differences between the widths of the $m_{\mathrm{s}}= \pm 3 / 2$ and the $m_{\mathrm{s}}=$ $\pm^{1} / 2$ can be used for the same purpose. The combined difference between the widths of the two outer lines and those of the two inner lines is equal to $\pi^{-1}(4 c)$ (eq 13). The values of $c$ for $C_{18}$ and $\mathrm{C}_{21}$ thus determined are given in Table IV. The coupled ${ }^{13} \mathrm{C}$ signals for methyl $\mathrm{C}_{19}$ was not well resolved from the other methylene signals. Therefore, the DLB for methyl $\mathrm{C}_{19}$ could not be determined.

${ }^{13} \mathrm{C} T_{1}$ and NOE at Several Magnetic Fields. The ${ }^{13} \mathrm{C} T_{1}$ and NOE measured at several magnetic fields $(2.1-9.4 \mathrm{~T})$ for both systems exhibit a strong magnetic field dependence (Tables II and III), which is characteristic of most of the micellar solutions studied at different magnetic fields ${ }^{31,32}$ and of many macromolecules such as proteins ${ }^{33}$ and nucleic acids. ${ }^{34}$ The field dependence of the

(31) For example: Lindman, B.; Ahnas, T.; Söderman, O.; Walderhaug, H.; Rapacki, K.; Stilbs, P. Faraday Discuss. Chem. Soc. 1983, 76, 317.

(32) (a) Walderhaug, H.; Söderman, O.; Stilbs, P. J. Phys. Chem. 1984, 88, 1655. (b) Söderman, O.; Henriksson, U.; Olsson, U. J. Phys. Chem. 1987, $91,116$.
TABLE IV: Order Parameter, S, and Correlation Times $\tau_{c}^{s}$ and $\tau_{c}{ }^{\prime}$ Derived from the Analysis of ${ }^{13} \mathrm{C} T_{1}$ and NOE Results and the Calculated and Experimental DLB Effects

\begin{tabular}{cccccl}
\hline $\mathrm{C}$ no. & $\mathrm{S}$ & $\tau_{\mathrm{c}}^{\mathrm{s}}, \mathrm{ns}$ & $\tau_{\mathrm{c}}^{\mathrm{f}}, \mathrm{ps}$ & $c^{\text {calcd } a}, \mathrm{~s}^{-1}$ & $c^{\text {expll } a} \mathrm{~s}^{-1}$ \\
\hline $\mathrm{C}_{18}$ & $0.21 \pm 0.01$ & & $10 \pm 1$ & $3.7 \pm 0.5$ & $3.6 \pm 1$ \\
$\mathrm{C}_{21}$ & $0.22 \pm 0.01$ & $4.3 \pm 0.5^{b}$ & $20 \pm 1$ & $4.0 \pm 0.5$ & $2.5 \pm 1$ \\
$\mathrm{C}_{19}$ & $0.23 \pm 0.01$ & & $12 \pm 2$ & $3.8 \pm 0.5$ & $c$
\end{tabular}

${ }^{a}$ Calculated for and measured at $B_{0}=7.0 \mathrm{~T}$. ${ }^{b} \tau_{\mathrm{c}}{ }^{\mathrm{s}}$ is the same for all the methyl groups according to the model. ${ }^{c}$ Not measurable due to overlap with other signals.

relaxation times and NOE has been treated by Wennerström et al. ${ }^{12}$ in the "two-step" model and by Lipari and Szabo ${ }^{13}$ in the "model free" approach. Similar forms of the two-step spectral densities have also been suggested by Freed and co-workers for anisotropic systems. ${ }^{35}$ In these models, the dynamics of the molecular aggregates or the macromolecules is described by an isotropic slow reorientation of the whole molecule (or aggregate) and a fast, and slightly anisotropic, internal reorientation of the segment of the molecule of interest. In the case of molecular aggregates, such as micelles, the slow isotropic motion also contains the contribution from diffusion of molecules along the surface of the aggregates. The formalisms thus allow the determination of the correlation times of the two motions and an order parameter that describes the time-averaged angular restriction of the fast internal motion. The ${ }^{13} \mathrm{C} T_{1}$ and NOE according to these models are given by ${ }^{12,13}$

$$
\begin{aligned}
& \left(T_{1 i}\right)^{-1}= \\
& \frac{N_{i}}{20}\left[\frac{\gamma_{\mathrm{H}} \gamma_{\mathrm{C}} \hbar}{r^{3}}\right]^{2}\left[J\left(\omega_{\mathrm{H}}-\omega_{\mathrm{C}}\right)+3 J\left(\omega_{\mathrm{C}}\right)+6 J\left(\omega_{\mathrm{H}}+\omega_{\mathrm{C}}\right)\right] \\
& \quad \eta_{i}=1 / 20 \gamma_{\mathrm{H}}^{3} \gamma_{\mathrm{C}}\left(\hbar / r^{3}\right)^{2}\left(N_{i} T_{1 i}\right)\left[6 J\left(\omega_{\mathrm{H}}+\omega_{\mathrm{C}}\right)-J\left(\omega_{\mathrm{H}}-\omega_{\mathrm{C}}\right)\right]
\end{aligned}
$$

Here, $\gamma_{H}$ and $\gamma_{C}$ represent the magnetogyric ratios of proton and carbon, respectively, $r$ denotes the $\mathrm{C}-\mathrm{H}$ bond distance, which is assumed to be $1.09 \AA$ in the present calculation, and $\eta_{i}$ is the NOE. The $J(\omega)$ values are the various reduced spectral densities, $\omega_{C}$ and $\omega_{\mathbf{H}}$ are the Larmor frequencies of carbon and proton at the actual magnetic field, and $\hbar$ is the reduced Planck's constant. $N_{i}$ is the number of protons directly bonded to carbon $i$.

The reduced spectral densities, $J(\omega)$, in these models are given by

$$
J(\omega)=\left(1-S^{2}\right) 2{\tau_{c}}^{f}+S^{2}\left(\frac{2 \tau_{c}^{s}}{1+\left(\omega \tau_{c}^{s}\right)^{2}}\right)
$$

Here $\tau_{c}{ }^{s}$ is the correlation time for the slow isotropic motion; $\tau_{c}{ }^{t}$ is the corrleation time for the fast local motion, which has to be sufficiently fast to fall in the extreme narrowing limit $\left(\omega_{0} \tau_{c} \ll\right.$ 1) for the models to be valid. $S$ is the order parameter for the $\mathrm{C}-\mathrm{H}$ bonds. In eq 21 and 22 , the DD relaxation between the ${ }^{13} \mathrm{C}$ and the attached proton nuclei is assumed to be the only significant mechanism. From the magnitude of the CSA determined in this study (see the next section), it is estimated that the CSA contribution to the spin-lattice relaxation is still relatively minor $(1-2 \%)$ even at $B_{0}=9.4 \mathrm{~T}$. Therefore, the use of eq 21 and 22 is valid.

Determination of $\tau_{\mathrm{c}}{ }_{\mathrm{c}}^{\mathrm{s}}, \tau_{\mathrm{c}}{ }^{\mathrm{f}}$, and $S$. Thus in studies involving the ${ }^{13} \mathrm{C} T_{1}$ and NOE of a number of carbon segments at several magnetic fields, the individual $\tau_{\mathrm{c}}{ }_{\mathrm{f}}$ and the order parameter, $\mathrm{S}$, for each segment, and a common slow correlation time, $\tau_{\mathrm{c}}{ }^{\mathrm{s}}$, can be determined from the experimental data by least-squares analysis. Alternatively, if the slow correlation time can be determined from

(33) For example: Henry, G. D.; Weiner, J. H.; Sykes, B. D. Biochemistry $1986,25,590$.

(34) For example: Schmidt, P. G.; Sierzputowska-Gracz, H.; Agris, P. F. Biochemistry 1987, 26, 8529 .

(35) Polnaszek, C. F.; Freed, J. H. J. Phys. Chem. 1975, 79, 2283. Freed, J. H. J. Chem. Phys. 1977, 66, 4183. 
independent means, the other two parameters, $\tau_{c}{ }_{c}$ and $S$, can be extracted from $T_{1}$ at two magnetic fields or from $T_{1}$ and NOE at a single magnetic field, assuming the two-step model (eq 23) is applicable. However, it is prudent, in most cases, to use data at more than one or two magnetic fields to ascertain whether the model is valid or not for the system under investigation.

The ${ }^{13} \mathrm{C} T_{1}$ and NOE data for the sodium deoxycholate micellar solution spanned a magnetic field range of 2.1-9.4 T. Thus, by use of a least-squares routine, the correlation times and the order parameter for methyl carbons 18,19 , and 21 were determined from the data presented in Table III. The correlation times and order parameter (Table IV) were then used in turn to calculate the $b$ and $c$ terms in the DLB equations, utilizing the relationship between $S$ and $S_{C_{3}}$ (eq 18). Good agreement between the values of the $c$ terms thus calculated and the experimental values was obtained (Table IV). The calculation also showed that the $b$ term for all the methyls is indeed too small $\left(\sim 0.3 \mathrm{~s}^{-1}\right)$ to be observed. The good agreement between the $T_{1}$ and NOE results on one hand and the DLB results on the other strongly indicates that the two-step model is applicable to the sodium deoxycholate micellar system. In such cases, the DLB and the multifield relaxation and NOE results can be combined to make a more unambiguous determination of the order parameter and the correlation times.

Previous studies of the deoxycholate system indicated that the surfactant forms spherical micelles. ${ }^{36}$ The derived $\tau_{c}{ }^{s}$ indicated that the aggregates are relatively small. These properties probably account for the applicability of the two-step model for this system. The present results are in close agreement with a recent work based on ${ }^{1} \mathrm{H}$ two-quantum relaxation of the methyl signals. ${ }^{37}$ In the present study, the order parameters for all the methyl $\mathrm{C}-\mathrm{H}$ were found to be approximately 0.2 , which implies that the order parameter $S_{C_{3}}$ for these methyl groups should be about 0.6. That is, there is rather limited wobbling motion for these methyl $C_{3}$ axes, which are attached to a rather rigid steroid backbone. However, in ref $37, S_{C_{3}}$ was found to be close to 1 for all the methyls. Otherwise, the agreement between the results of ref 37 and the present work, particularly in the slow correlation time, is very good.

The results for the CTAB/Nasal viscoelastic micellar system presented a rather different picture. The ${ }^{13} \mathrm{C} T_{1}$ and NOE data were limited to only two magnetic fields, i.e., 7.0 and $9.4 \mathrm{~T}$. From the magnetic field dependence of the $T_{1}$ and NOE, a "slow" motion faster than $10^{-7} \mathrm{~s}$ was predicted on the basis of the two-step model. Least-squares analysis of the $T_{1}$ and NOE data showed that $\tau_{\mathrm{c}}{ }^{\mathrm{s}}$ should be $(2.0-2.5) \times 10^{-8} \mathrm{~s}$ for the two samples studied at several temperatures. However, the resultant order parameter for the $N$-methyl $\mathrm{C}-\mathrm{H}, \mathrm{S}$, was found to range from 0.25 to 0.39 . These values for $\mathrm{S}$ are unreasonably high and are contradictory to the observed order parameter for the C-D bonds in $C_{n} N\left(C_{3}\right)_{3}$ in lamellar and hexagonal liquid crystalline phases. ${ }^{38}$ In these phases, the order parameters $S_{C D}$, which should be practically equivalent to $S_{C H}$ (and $S$ in the present case), was generally found to be $\sim 0.037 \pm 0.005$. If this value is used for $S$, then $S_{C_{3}}$ is $\sim 0.11$. From the experimental DLB results, the slow correlation time, $\tau_{c}^{\mathrm{s}}$, was found to be $1.5-2.4 \mu \mathrm{s}$, which is 2 orders of magnitude longer than that suggested by the ${ }^{13} \mathrm{C} T_{1}$ and NOE results. However, the results determined from DLB assuming $S \sim 0.037$ are consistent with the results of several other investigations.

1. From ${ }^{1} \mathrm{H}$ line-shape measurements, Olsson et al. ${ }^{39}$ concluded that the $\tau_{\mathrm{c}}{ }^{\mathrm{s}}$ for viscoelastic micelles based on CTAB was in the microsecond range for concentrations corresponding to those in this work.

2. The CSA thus determined (from the experimental values of the $b$ term) assuming $\mathrm{S}=0.037$ and $\mathrm{S}_{C_{3}}=0.11$ was approximately $-60 \mathrm{ppm}$. Thus, $\mathrm{S}_{C_{3}} \Delta \sigma \approx 6 \mathrm{ppm}$, a value consistent with the observed residual $\mathrm{CSA}$ for the $N$-methyl ${ }^{13} \mathrm{C}$ in $\mathrm{CTAB}$ in hexagonal and lamellar liquid crystal phases. ${ }^{40}$

(36) Small, D. M. The Bile Acids; Plenum: New York, 1971.

(37) Kay, L. E.; Prestegard, J. H. J. Am. Chem. Soc. 1987, 109, 3829

(38) Olsson, U.; Söderman, O., unpublished results.

(39) Olsson, U.; Söderman, O.; Guering, P. J. Phys. Chem. 1986, 90, 5223.

(40) Henriksson, U.; Klasson, T. Finn. Chem. Lett. 1982, 139.
3. The observed DLB for the ${ }^{13} \mathrm{C}$ multiplet of the $N$-methyl group in CTAB was about $20 \mathrm{~Hz}$ for $\Delta v\left({ }^{3} / 2,1 / 2\right)$ in the viscoelastic solutions (Table I) but was not measurable $(<0.3 \mathrm{~Hz})$ in the "normal" micellar solutions. Since the order parameters, which reflect only the effects of the fast local motion, should be similar in these two types of solutions, there is then a difference of at least 2 orders of magnitude between the slow correlation times for the two types of systems. According to previous studies of the normal micellar systems of dodecyl- and hexadecyltrimethylammonium chlorides (DOTAC and CTAC), ${ }^{32}$ the slow correlation time for such micellar aggregates was found to be $(1.5-3) \times 10^{-9} \mathrm{~s}$ by ${ }^{13} \mathrm{C}$ multifield relaxation experiments and (4-8) $\times 10^{-9} \mathrm{~s}$ by a ${ }^{2} \mathrm{H}$ mulifield study. Therefore, $\tau_{c}^{s}$ for the viscoelastic micelles should be longer than $10^{-7} \mathrm{~s}$. The value of $(2.0-2.5) \times 10^{-8} \mathrm{~s}$ for $\tau_{\mathrm{c}}^{\mathrm{s}}$ for the viscoelastic system as suggested by the ${ }^{13} \mathrm{C} T_{1}$ and NOE data based on the two-step model is clearly too short to account for the difference in the observed DLB in the two systems.

On the basis of the above comparisons, it was concluded that the calculation of $\tau_{c}$ f from DLB based on the value of $S$ taken from ${ }^{2} \mathrm{H}$ splittings of the $N$-methyl deuterons of CTAB ( $N$-methyl deuteriated) in liquid crystalline phases was correct. Then the discrepancy between the DLB results, which primarily arise from the spectral densities at zero frequency, and the ${ }^{13} \mathrm{C} T_{1}$ and NOE results, which sample the spectral density at Larmor frequencies of $10^{8}-10^{9} \mathrm{~Hz}$, is due to the existence of motion(s) of an intermediate $\left(10^{-7}-10^{-8} \mathrm{~s}\right)$ time scale. In other words, the simple "two-step" model cannot be used to described the dynamics of the CTAB/Nasal viscoelastic micellar system. The intermediate motion(s) causes the field dependence of the ${ }^{13} \mathrm{C} T_{1}$ and NOE (a single slow motion with a correlation time of $10^{-6} \mathrm{~s}$ would have resulted in field-independent ${ }^{13} \mathrm{C} T_{1}$ and NOE at the magnetic fields of our measurements), while the DLB measures the effects of the slowest motion (in this case $>10^{-6} \mathrm{~s}$ ). The existence of complex motions and the breakdown of the simple "two-step" model for this system are hardly surprising, ${ }^{39}$ since the system has been shown to form rodlike micelles that are probably quite flexible. ${ }^{22,39}$ Furthermore, many recent studies utilizing ${ }^{13} \mathrm{C} T_{1}$ and NOE and ${ }^{2} \mathrm{H} T_{1}$ and $T_{2}$ measurements demonstrated that there are many surfactant systems that exhibit more complicated dynamics than that described by the "two-step" model. ${ }^{41,42} \mathrm{~A}$ DLB and ${ }^{13} \mathrm{C} T_{1}$ investigation of the cubic liquid crystal phase $\left(I_{2}\right)$ of DOTAC/water system ${ }^{43}$ also revealed discrepancies between the DLB and the ${ }^{13} \mathrm{C} T_{1}$ results if the two-step model is applied. The existence of more complicated motion(s) in this system was confirmed by ${ }^{2} \mathrm{H} T_{1}$ and $T_{2}$ results. ${ }^{44}$

Since the correlation time for the slowest motion $\left(\sim 10^{-6} \mathrm{~s}\right)$ for the CTAB/Nasal system was determined from DLB, it is in principle possible to use an extension of the "two-step" model to evaluate the correlation times for the fast internal motion and the intermediate motion(s). Such attempts have been made for several isotropic surfactant systems where more than one "slow" motions were implicated, for example, a "three-step" model of the form ${ }^{42}$

$J(\omega)=$

$$
2\left(1-S_{1}^{2}\right) \tau_{c}^{f}+\left(S_{1}{ }^{2}-S_{2}{ }^{2}\right) \frac{2 \tau_{c}^{i}}{1+\left(\omega \tau_{c}^{i}\right)^{2}}+S_{2}{ }^{2} \frac{2 \tau_{c}^{s}}{1+\left(\omega \tau_{c}^{s}\right)^{2}}
$$

or ${ }^{41}$

$$
J(\omega)=2\left(1-\mathrm{S}^{2}\right) \tau_{\mathrm{c}}{ }^{\mathrm{f}}+\mathrm{S}^{2}\left(\frac{3}{4} \frac{2 \tau_{\mathrm{S}}}{1+\left(\omega \tau_{\mathrm{S}}\right)^{2}}+\frac{1}{4} \frac{2 \tau_{\perp}}{1+\left(\omega \tau_{\perp}\right)^{2}}\right)
$$

In the first equation, $\tau_{\mathrm{c}}{ }_{\mathrm{i}}$ stands for the correlation time for the intermediate motion, and $S_{1}$ and $S_{2}$ are the order parameters after averaging over the fast motion and the intermediate motion,

(41) Söderman, O.; Walderhaug, H.; Henriksson, U.; Stilbs, P. J. Phys. Chem. 1985, 89, 3693. Nery, H.; Söderman, O.; Canet, D.; Walderhaug, H.; Lindman, B. J. Phys. Chem. 1986, 90, 5802.

(42) Carnali, J.; Lindman, B.; Söderman, O.; Walderhaug, H. Langmuir 1986, 2, 51 .

(43) Wong, T. C., unpublished results.

(44) Henriksson, U.; Stilbs, P.; Söderman, O., unpublished results. 
respectively. In the second equation, the spectral density for the slow motions is the specific result of the solution of the rotational diffusion equation for a symmetric top, ${ }^{45}$ where $\tau_{\perp}$ and $\tau_{\mathrm{S}}$ are correlation times for the rotation about the long axis of the rod and the end-over-end rotation, respectively. The end-over-end tumbling generally occurs at the slowest time scale. However, since there was no direct knowledge of the spectral densities at the intermediate frequencies and since the motion(s) of the viscoelastic micellar aggregates in this frequency range are likely to be rather complex, we do not feel that a quantitative evaluation of the correlation times based on the present data is worthwhile.

The results of the calculation for the CTAB/Nasal viscoelastic system assuming $\mathrm{S}=0.037$ is given in Table $\mathrm{I}$. The chemical shift anisotropy for the $N$-methyl ${ }^{13} \mathrm{C}$ calculated from the $b$ term was found to lie within the range -56 to $-75 \mathrm{ppm}$. However, since the measurements at the higher temperature range $\left(47-56^{\circ} \mathrm{C}\right)$ are more reliable, the more reliable value of the CSA should range from -56 to $-65 \mathrm{ppm}$. The deduced value of the CSA of about $-60 \mathrm{ppm}$ for the $N$-methyl ${ }^{13} \mathrm{C}$ is quite large compared with those of $C$-methyl groups (typically from -20 to $-40 \mathrm{ppm}$ ) but is of approximately the same magnitude of those of $O$-methyl groups (typically from -50 to $-80 \mathrm{ppm}$ ). ${ }^{27}$

The DLB enables one to determine the sign of the CSA provided the absolute sign of $1 J_{\mathrm{CH}}$ is known., 1,3,4,7 The negative sign of $\sigma_{\text {H }}$ - $\sigma_{\perp}$ determined in this study is consistent with those of the $C$-methyl and $O$-methyl ${ }^{13} \mathrm{C} .{ }^{27}$ It has been shown that the chemical shielding tensors of some methyl ${ }^{13} \mathrm{C}$, particularly those of $O$ methyl groups, deviate slightly from cylindrical symmetry. ${ }^{27}$ In such cases, then, $\sigma_{1}-\sigma_{\perp}$ should be replaced by $\sigma_{\|}-1 / 2\left(\sigma_{a a}+\right.$ $\left.\sigma_{b b}\right)$, where $a$ and $b$ denote the two principal axes of the CSA tensor perpendicular to the parallel axis. If the CSA of the methyl ${ }^{13} \mathrm{C}$ is known from an independent measurement, the consistency of the calculation described above and the value of $\tau_{c}{ }^{s}$ can be checked and ascertained. In the present case, the CSA of the $N$-methyl group is not known. Because of the complications caused by the quadrupole moment of the neighboring ${ }^{14} \mathrm{~N}$, as far as we know, no information on the CSA of $N$-methyl carbon is available in the literature.

\section{Discussions}

In this work, the theory of differential line broadening of the methyl multiplet in systems where there is a slow overall reorientation and fast, and slightly anisotropic, internal motions has been derived on the basis of the solution of the stochastic Liouville equation. Several important categories of systems-molecular aggregates such as micelles, microemulsions, and vesicles, and biomacromolecules such as nucleic acids and proteins-fall into this category. Indeed, DLB in the methyl group has so far been observed, in addition to the present study of micellar solutions, in transfer RNA ${ }^{11}$ and in a cubic liquid crystal phase. ${ }^{43}$ The DLB in a methyl group is unique in that it is in principle possible to determine both the slow correlation time and the chemical shift anisotropy from the differences between the various lines of the multiplet. In practice, the applicability of this effect can be determined only with further work on other real systems.

The determination of the $\tau_{c}$ s from DLB is particularly useful in the study of the dynamics of macromolecules and molecular aggregates. It can provide an independent comparison with the

(45) Huntress, W. T. Adv. Magn. Reson. 1970, 4, 1. values determined from multifield $T_{1}$ and NOE studies. In the two systems studied in this work, it is demonstrated that the DLB results can be combined with the multifield $T_{1}$ and NOE measurements to determine the correlation times and order parameters for systems to which the two-step model is applicable. On the other hand, the DLB techniques can also be used as a diagnosis for the breakdown of the two-step model in more complex systems, such as in the viscoelastic micelles. In such a case, DLB provides the measurement of the slowest correlation time, which is frequently inaccessible from measurements at higher frequencies.

For methyl groups of known ${ }^{13} \mathrm{C} \mathrm{CSA}$, the various line-width differences can be used to provide a further check of the internal consistency of the value of $\tau_{c}{ }^{\mathrm{s}}$ determined (the $b$ and $c$ terms). In studies in which the molecular dynamics in the main objective of the investigation, this is probably the preferred approach. On the other hand, in a situation where the CSA is not known or cannot be independently measured, as in the present case, the DLB provides a reasonable way to estimate the CSA.

Recently, there have been revived interests in the differential relaxation rates in coupled spin systems. ${ }^{3-7,37}$ Higher magnetic fields used in NMR and the increasing size of the molecules studied increase dramatically the likelihood of observing this type of phenomenon. A study closely related to DLB by Jaccard et al. demonstrated the observation of longitudinal two-spin order $2 I_{\mathrm{Z}} S_{\mathrm{Z}}$ due to the interference between CSA and dipolar interactions. ${ }^{46}$ Differential relaxation rates not only provide valuable information on the molecular dynamics and chemical shift anisotropy as demonstrated in this and several previous work $\mathrm{k}^{3-7}$ but also are responsible for some of the previously unexpected observations in two-dimensional (2D) NMR spectroscopy of macromolecules in which the rate of the molecular reorientation is not in the extreme narrowing limit. Prominent examples are the appearance of isolated methyl signals in multiple-quantum filtered 2D experiments ${ }^{37,47-49}$ and relaxation-allowed coherence transfer between spins not scalarly coupled to each other. ${ }^{50}$ Therefore, it appears that relaxation in coupled spin systems will attract much further research effort.

Acknowledgment. This work is partially supported by an NIH Biomedical Research Support Grant. The NT-300 spectrometer at the University of Missouri was purchased through a grant from the National Science Foundation (PCM-8115599). The use of the $400-\mathrm{MHz}$ spectrometer at the NMRFAM at the University of Wisconsin, Madison, is gratefully acknowledged. T.C.W. would like to thank Dr. Hanna Gracz for communicating the observation of DLB in transfer RNA to him and Professor T. C. Farrar for stimulating discussions. L.P.H. thanks the National Science Council of the Republic of China for a Travelling Grant and the University of Missouri for hospitality during his stay, Dr. Olle Söderman's valuable suggestions concerning the viscoelastic micelles are greatly acknowledged.

Registry No. Nasal, 54-21-7; CTAB, 57-09-0; sodium deoxycholate, 302-95-4.

(46) Jaccard, G.; Wimperis, S.; Bodenhausen, G. Chem. Phys. Lett. 1987, 138,601 .

(47) Muller, N.; Bodenhausen, G.; Wuthrich, K.; Ernst, R. R. J. Magn. Reson. 1985, 65, 531 .

(48) Muller, N.; Ernst, R. R.; Wuthrich, K. J. Am. Chem. Soc. 1986, 108, 6482.

(49) Rance, M.; Wright, P. E. Chem. Phys. Lett. 1986, 124, 572

(50) Wimperis, S.; Bodenhausen, G. Chem. Phys. Lett. 1987, 140, 41

(51) Söderman, O. J. Magn. Reson. 1986, 68, 296. 